\title{
Premixed Combustion in Boundary Layers for Moderate Values of the Zeldovich Numbers
}

\author{
F. MENDEZ \\ C. TREVIÑO \\ A. LIÑÁN
}

\begin{abstract}
The classical problem of the ignition and flame generation of combustible premixed gases by a hot flat plate is analyzed by asymptotic techniques for moderate vatues of the Zeldovich numbers. For temperatures of the plate smaller than the adiabatic flame temperature it was found that the corrections to the zeroth-order solution (Zeldovich approximation) are very important for finite Zeldovich numbers. The asymptotic analysis was carried out with the inclusion of reactant consumption showing different responses depending if the reactant consumption parameter is larger or smaller than a critical one. For tempcratures of the plate larger than the adiabatic flame temperatures (that is, the reactant consumption parameter larger than the critical one), the ignition is not characterized by a thermal runaway and a smooth transition from frozen to equilibrium flow is obtained. The evolution towards the premixed flame formation is analyzed and the flame position and flame temperature are obtained.
\end{abstract}

\section{INTRODUCTION}

The ignition process of combustible premixed gases by hot surfaces (plate) have received much attention in the literature in the past decades. Sharma and Sirignano (1970) numerically integrated the governing equations. They used as the ignition criterion the adiabaticity condition at the plate. Berman and Ryazantsev (1978) using asymptotic techniques analyzed the ignition process for very high Zeldovich numbers. They found the leading term of the asymptotic expansion for the critical distance for ignition which coincides with the adiabaticity criterion. The reactant consumption effects have been included. Law and Law (1979) made an analysis in the same line, comparing their numerical results with the leading term in the asymptotic expansion for the ignition distance. They found that the asymptotic value of the Damköhler number for ignition underestimates up to half of the value of that obtained through numerical methods. In a later paper Law and Law (1981) showed better agreements when considering the reactant consumption effects. For large but finite Zeldovich numbers of the chemical reactions, the corrections to the leading term can be very important, as was shown by Liñán and Williams (1979) for the transient ignition of the reactive solid. The physical explanation for this is the following. When approaching the critical ignition conditions, the longitudinal temperature gradients in the gas causes an increase of the heat losses through the boundary layer. This makes sure that the last part of the ignition process is delayed by a quantity of the same order as the leading term for finite Zeldovich numbers. Kapila (1980, 1981) has shown that after reaching the ignition condition, the unsteady effects delay the further development of the premixed flame in a very short time scale which ends in a hot spot generation growing in intensity in an exponentially small time scale. Then, this hot spot transforms into a deflagration wave. In the analysis made by Kapila, similarity between concentration and temperature was assumed. 
The main objective of this paper is, with the inclusion of the reactant consumption effects, to derive the correction for the critical Damköhler number for ignition and to study the further evolution of a premixed flame in this boundary layer flow.

\section{PROBLEM FORMULATION}

The physical model analyzed in this paper is the following. A combustible gaseous mixture flows over a flat plate at a high temperature $\bar{T}_{p}$. The free stream is associated with a gas velocity $u_{\infty}$, temperature $\bar{T}_{\infty}$ and species mass concentration $\bar{Y}_{i \infty}$. The chemical reaction assumed for the gas-phase is one step and irreversible of the form

$$
\nu_{F}|F|+\nu_{0}|O| \rightarrow \nu_{p}|P|,
$$

where $\nu_{i}$ are the stoichiometric coefficients with $i=F, O, P$ for the fuel, oxidizer and product, respectively. The symbol $[i]$ is identified with the chemical formula of the specie $i$. The fuel generation through the chemical reaction is assumed to be given by the Arrhenius kinetics of the form

$$
w_{F}=-B v_{F} W_{F} \bar{Y}_{F} \rho \exp \left(-\frac{E}{R \bar{T}}\right),
$$

where $w_{F}$ is the mass production of fuel for unit volume and time, $B$ and $E$ are the frequency factor and the activation energy of the chemical reaction, respectively. $W_{i}$ is the molecular weight of the specie $i . \bar{Y}_{i}$ and $\bar{T}$ are the mass concentration of the specie $i$ and the temperature of the gas, respectively. In Eq. (2) it is assumed for simplicity a lean mixture and a unity reaction order for the fuel. Therefore, the mass concentration of the oxidant is assumed to be almost constant. The reactive governing equations for this boundary layer flow configuration are well known (see, for example, Sharma and Sirignano, 1970; Treviño and Sen, 1980; Williams, 1985) and will not be repeated here. We introduce the following non-dimensional dependent variables

$$
T=\frac{C_{p}\left(\bar{T}-\bar{T}_{p}\right)}{Q \bar{Y}_{F \infty}}, \quad Y=\frac{\bar{Y}_{F}}{\bar{Y}_{F \infty}}, \quad f=\frac{\psi}{\sqrt{ }\left(2 \mu_{\infty} \rho_{\infty} u_{\infty} \bar{x}\right)},
$$

where $C_{p}$ is the specific heat at constant pressure, $Q$ is the heat of reaction for unit mass of fuel consumed, $\rho$ and $\mu$ are the density and viscosity coefficients, respectively, and $\psi$ and $f$ are the stream function dimensional and non-dimensional, respectively. Introducing the following independent variables

$$
\xi=\frac{\bar{x}}{L}, \quad \eta=\sqrt{\left(\frac{u_{\infty}}{2 \rho_{\infty} \mu_{\infty} \bar{x}}\right)} \int_{0}^{\bar{y}} \rho\left(\bar{x}, \bar{y}^{\prime}\right) d \bar{y}^{\prime},
$$

where $\bar{x}$ and $\bar{y}$ are the longitudinal and transversal Cartesian coordinates with the leading edge of the plate as the origin and $L$ is the length of the plate, the nondimensional governing equations transform to

$$
\begin{gathered}
\mathscr{L}\left(1, \frac{d f}{d \eta}\right)=0 \\
-\mathscr{L}(P r, T)=+\mathscr{L}(S c, Y)=\frac{2 \bar{B} L}{u_{\infty}} \exp \left(-\frac{\bar{T}_{a}}{\bar{T}_{p}}\right) \xi Y \exp \left(\frac{Z T}{1+\varepsilon Z T}\right),
\end{gathered}
$$


where the operator $\mathscr{L}$ is defined as

$$
\mathscr{L}(X, \psi)=\frac{1}{X} \frac{\partial^{2} \psi}{\partial \eta^{2}}+f \frac{\partial \psi}{\partial \eta}-2 \xi \frac{d f}{\partial \eta} \frac{\partial \psi}{\partial \xi}
$$

and $\bar{B}=B v_{F} W_{F}$.

In the above equations it is assumed that the Prandtl number, $\operatorname{Pr}=\mu C_{p} / \lambda$, the Schmidt number, $S c=\mu / \rho D$, as well as the product $\rho \mu$ are constants. $D$ is the diffusivity coefficient of the fuel and $\lambda$ is the thermal conductivity. $\bar{T}_{a}$ corresponds to the activation temperature $E / R . Z$ is the Zeldovich number and represents the ratio of the activation energy to the thermal energy and is given by

$$
Z=\frac{\widetilde{T}_{a} Q \tilde{Y}_{F \infty}}{C_{p} \tilde{T}_{p}^{2}}
$$

The parameter $\varepsilon$ is related to the inverse of the Zeldovich number and is given by

$$
\varepsilon=\frac{\bar{T}_{p}}{\bar{T}_{a}}=\frac{Q \bar{Y}_{F \infty}}{C_{p} \bar{T}_{p}} \cdot \frac{1}{Z} .
$$

In combustion the Zeldovich number can be assumed to be much larger than unity. The non-dimensional boundary conditions transform to:

$$
\begin{aligned}
& \text { at } \eta=0 ; \quad T=0 \text { and } \frac{\partial Y}{\partial \eta}=0, \\
& \text { at } \eta \rightarrow \infty ; T=\frac{C_{p}\left(\bar{T}_{\infty}-\bar{T}_{p}\right)}{Q \bar{Y}_{F \infty}}=T_{\infty} \text { and } Y=1 .
\end{aligned}
$$

Without the reaction term, the governing Eqs. (5), (6) and (10) have a self-similar solution. With the aid of the Lighthill approximation (Lighthill, 1950) for high Prandtl numbers (which in fact gives very good results for Prandtl number of order unity), the solution close to the wall is given by

and

$$
T_{f}=-\beta f^{\prime \prime}(0) \operatorname{Pr}^{1 / 3} \eta+O\left(\eta^{4}\right) \text { for } \quad \eta \rightarrow 0
$$

where

$$
Y=1
$$

$$
\beta=\frac{\left(\bar{T}_{p}-\bar{T}_{\infty}\right)}{Q \bar{Y}_{F \infty}} C_{p}=O(1)
$$

As shown by Eq. (11), the chemical reaction will take place only close to the plate for high Zeldovich numbers, due to the high sensitivity of the reaction rate of the temperature.

\section{ASYMPTOTIC SOLUTION}

\section{Reactive Zone}

For high Zeldovich numbers the gas-phase chemical reaction will only develop, in this ignition process, in regions close to the wall. In this reactive zone, an increase 
in the temperature above its frozen value of the order of the inverse of the Zeldovich number will produce an increase of order unity in the reaction rate. To study this ignition process we can introduce $\theta$ as:

$$
\theta=\left(T-T_{f}\right) Z
$$

Introducing the following stretched inner coordinate of the form

$$
\chi=Z \beta f^{\prime \prime}(0) \operatorname{Pr}^{1 / 3} \eta,
$$

the inner equations reduce to

$$
\begin{gathered}
\frac{\partial^{2} \theta}{\partial \chi^{2}}=-\frac{D Y \xi}{2} \exp \left[\frac{\theta-\chi}{1+\varepsilon(\theta-\chi)}\right]+\frac{2 \varepsilon^{3}}{\lambda^{3}}\left[\chi \xi \frac{\partial \theta}{\delta \xi}-\frac{1}{4} \chi^{2} \frac{\partial \theta}{\partial \chi}\right], \\
\frac{\partial^{2} Y}{\partial \chi^{2}}=\frac{D Y \xi}{2} \frac{\varepsilon \beta}{\lambda} L_{e} \exp \left[\frac{\theta-\chi}{1+\varepsilon(\theta-\chi)}\right]+2 \frac{\varepsilon^{4}}{\lambda^{4}} \beta L_{\theta}\left[\chi \xi \frac{\partial Y}{\partial \xi}-\frac{1}{4} \chi^{2} \frac{\partial Y}{\partial \chi}\right],
\end{gathered}
$$

where $D$ is the Damköhler number and is defined as

$$
D=\frac{2 \vec{B}}{u_{\infty}} \frac{L P_{r} 1^{1 / 3} \varepsilon \exp \left(-\bar{T}_{a} / \widetilde{T}_{p}\right)}{\left[\beta f^{\prime \prime}(0)\right]^{2}},
$$

$L_{e}$ is the Lewis number defined as $L_{e}=S c / P_{r}$ and $\lambda$ is a temperature parameter of order unity given by

$$
\lambda=\frac{\bar{T}_{p}-\bar{T}_{\infty}}{\bar{T}_{p}} .
$$

Equations (15) and (16) are to be solved with the boundary conditions at the plate $(X=0)$ given by

$$
\text { at } \chi=0 ; \theta=0 \text { and } \frac{\partial Y}{\partial X}=0 .
$$

The other two boundary conditions at the edge of the reactive layer come from the matching with the non-reactive outer boundary layer flow. This is going to be obtained later. Equation (15) shows that the convective terms are of order $\varepsilon^{3}$ and then its effect can be omitted at least until the last part of the ignition regime, where the temperature and concentration gradients are large enough.

It is convenient to redefine the inner variables of the form

and

$$
\psi=\theta-\chi
$$

$$
\phi=D Y .
$$

With the aid of Eqs. (20), Eqs. (15) and (16) take the form

$$
\frac{\partial^{2} \psi}{\partial \chi^{2}}=-\frac{\phi \xi}{2} \exp (\psi)\left[1-\varepsilon \psi^{2}\right],
$$




$$
\frac{1}{\gamma} \frac{\partial^{2} \phi}{\partial \chi^{2}}=\frac{\phi \xi}{2} \exp (\psi)\left[1-\varepsilon \psi^{2}\right]
$$

where the terms of order $\varepsilon^{2}$ are dropped out. Here $\gamma$ is a small number and is defined as

$$
\gamma=L_{\ominus} \frac{D \varepsilon}{\lambda} \beta .
$$

The boundary conditions at the plate surface then take the form

$$
\text { at } \chi=0 ; \psi=0 \text { and } \frac{\partial \phi}{\partial \chi}=0 \text {. }
$$

\section{Non-Reactive Zone Equations}

In this outer zone, the chemical reaction is exponentially small, and the solution to the inert problem given by Eqs. (6) without the reaction term is using the Lighthill approximation (Lighthill, 1950)

$$
\left(\frac{\partial \theta_{e}}{\partial \eta}\right)_{0}=-f^{\prime \prime}(0) \operatorname{Pr}^{1 / 3} \int_{0}^{\xi} \frac{d \theta_{e}^{\prime}}{d \xi^{\prime}}\left(\xi^{\prime}, 0\right) \frac{d \xi^{\prime}}{\left\{1-\left(\xi^{\prime} \mid \xi^{3 / 4}\right\}^{1 / 3}\right.},
$$

and

$$
\left(\frac{\partial \phi_{e}}{\partial \eta}\right)_{0}=-f^{\prime \prime}(0) S c^{1 / 3} \int_{0}^{\xi} \frac{d \phi_{e}^{\prime}}{d \xi^{\prime}}\left(\xi^{\prime}, 0\right) \frac{d \xi^{\prime}}{\left\{1-\left(\xi^{\prime} \mid \xi\right)^{3 / 4}\right\}^{1 / 3}},
$$

where the gradients are evaluated not at the surface but at the edge of the reactive zone. The frozen leading edge conditions lead to $\theta_{e}(0, \eta)=0$ and $\phi_{e}(0, \eta)=0$. For this reason the jump conditions at the leading edge are left out in Eqs. (25) and (26).

\section{Reactive Zone Solution}

Due to the impermeable condition at the plate $(\partial \phi / \partial X=0)$ it is convenient to assume a solution of the form

$$
\phi=\phi_{\delta}(\xi)+\varepsilon \phi_{1}(\xi, \chi)+\ldots
$$

for the fuel concentration. Introducing the relation (27) into Eqs. (21) and (22) leads to

$$
-\frac{\partial^{2} \psi}{\partial X^{2}}=\frac{\varepsilon}{\gamma} \frac{\partial^{2} \phi_{1}}{\partial X^{2}}=\frac{\phi_{s} \xi}{2} \exp (\psi)\left[1-\varepsilon \psi^{2}\right]-\frac{\varepsilon \phi_{1}}{2} \xi \exp (\psi),
$$

which is valid up to terms of order $\varepsilon^{2}$. A first integration of the energy Eq. (28) gives

$$
\begin{aligned}
\left(\frac{\partial \psi}{\partial X}\right)^{2}-q^{2}= & \phi_{8} \xi(1-2 \varepsilon)-\phi_{s} \xi \exp (\psi)\left[1-\varepsilon\left(\psi^{2}-2 \psi+2\right)\right] \\
& -\varepsilon \xi\left\{\exp (\psi) \phi_{1}-\phi_{1 p}+\frac{\gamma}{\varepsilon}[\exp (\psi)-1]-\frac{\gamma}{\varepsilon} q \int_{0}^{x} e^{\psi} d x\right\}
\end{aligned}
$$


where $q$ is the non-dimensional heat transfer at the plate

$$
q=\left(\frac{\partial \psi}{\partial X}\right)_{p}
$$

which in fact has the value of -1 for frozen flow. In Eq. (29) $\phi_{1 p}$ is the value of $\phi_{1}$ at the plate. At the edge of the reactive zone $(x \rightarrow \infty)$, from Eq. (29) we have

$$
\left.\frac{\partial \psi}{\partial X}\right|_{\infty} \sim-\sqrt{ }\left(\phi_{s} \xi+q^{2}\right)+\frac{\varepsilon \xi\left[\phi_{s}-\frac{1}{2}\left(\phi_{1 p}+\frac{\gamma}{\varepsilon}+\frac{\gamma}{\varepsilon} q \int_{0}^{x} e^{\psi} d x\right)\right]}{\sqrt{ }\left(\phi_{s} \xi+q^{2}\right)}+O\left(\varepsilon^{2}\right),
$$

where a formal expansion for small values of $\varepsilon$ has been made. Integrating Eq. (29) and evaluating this for high values of $\chi$, we obtain

$$
\begin{aligned}
\psi \sim\left\{-\sqrt{ }\left(\phi_{s} \xi+q^{2}\right)+\frac{\varepsilon \xi\left[\phi_{s}-\frac{1}{2}\left(\phi_{1 p}+\frac{\gamma}{\varepsilon}+\frac{\gamma}{\varepsilon} q \int_{0}^{x} e^{\psi \gamma} d x\right)\right]}{\sqrt{ }\left(\phi_{s} \xi+q^{2}\right)}\right\} x \\
+\ln \left\{\frac{4}{\phi_{s} \xi}\left(\phi_{s} \xi+q^{2}\right) \frac{\sqrt{ }\left(\phi_{s} \xi+q^{2}\right)+q}{\sqrt{ }\left(\phi_{s} \xi+q^{2}\right)-q}\right\}+O(\varepsilon) \text { as } x \rightarrow \infty,
\end{aligned}
$$

where the logarithmic term denotes the apparent temperature of the plate viewed from the frozen outer zone.

Subtracting the energy from the fuel concentration Eq. (28) for the inner zone, we obtain

$$
\frac{\partial^{2} \psi}{\partial X^{2}}+\frac{\varepsilon}{\gamma} \frac{\partial^{2} \phi_{1}}{\partial X^{2}}=0 .
$$

Integrating Eq. (33) and applying the boundary conditions at the plate, gives

$$
\frac{\partial \psi}{\partial \chi}+\frac{\varepsilon}{\gamma} \frac{\partial \phi_{1}}{\partial \chi}=q .
$$

The fuel concentration gradient at the edge of the reactive zone with the aid of Eq. (31) is then given by

$$
\frac{\partial \phi_{1}}{\partial X} \sim \frac{\gamma}{\varepsilon}\left[+\sqrt{ }\left(\phi_{s} \xi+q^{2}\right)\right]+O(\varepsilon) .
$$

Integration of Eq. (34) then gives

$$
\begin{aligned}
\phi_{1} \sim \phi_{1 p} & +\frac{\gamma}{\varepsilon}\left\{q \chi+\sqrt{ }\left(\phi_{s} \xi+q^{2}\right) \chi\right. \\
& \left.-\ln \left[\frac{4}{\phi_{s} \xi}\left(\phi_{s} \xi+q^{2}\right) \frac{\sqrt{ }\left(\phi_{s} \xi+q^{2}\right)+q}{\sqrt{ }\left(\phi_{s} \xi+q^{2}\right)-q}\right]\right\} \text { as } x \rightarrow \infty .
\end{aligned}
$$


It is convenient to have no apparent fuel concentration in this order (it can be absorbed in $\phi_{s}$ ). To do this, we put

$$
\phi_{1 p}=\frac{\gamma}{\varepsilon} \ln \left\{\frac{4}{\phi_{s} \xi}\left(\phi_{\varepsilon} \xi+q^{2}\right) \frac{\sqrt{ }\left(\phi_{\varepsilon} \xi+q^{2}\right)+q}{\sqrt{ }\left(\phi_{s} \xi+q^{2}\right)-q}\right\} .
$$

Matching requires that for the parametric limit in which $\varepsilon$ goes to zero, in an intermediate variable the relationship $\theta_{e}(\xi, \eta) \sim \theta(\xi, X)$ and $\phi_{e}(\xi, \eta) \sim \phi(\xi, X)$ must hold. These are given by

$$
\begin{aligned}
& 1-\sqrt{ }\left(\phi_{s} \xi+q^{2}\right)+\frac{\varepsilon \xi\left[\phi_{s}-\frac{1}{2}\left(\phi_{1 p}+\frac{\gamma}{\varepsilon}+\frac{\gamma}{\varepsilon} q \int_{0}^{\infty} e^{\psi} d x\right)\right]}{\sqrt{ }\left(\phi_{s} \xi+q^{2}\right)} \\
& \sim-\frac{\varepsilon}{\lambda} \int_{0}^{\xi} \frac{d \theta_{e}^{\prime}}{d \xi^{\prime}} \frac{d \xi^{\prime}}{\left\{1-\left(\xi^{\prime} / \xi\right)^{3 / 4}\right\}^{1 / 3}}
\end{aligned}
$$

and

$$
\begin{gathered}
-\alpha D\left\{q+\sqrt{ }\left(\phi_{s} \xi+q^{2}\right)-\frac{\varepsilon \xi\left[\phi_{s}-\frac{1}{2}\left(\phi_{1 p}+\frac{\gamma}{\varepsilon}+\frac{\gamma}{\varepsilon} q \int_{0}^{\infty} e^{\psi} d x\right)\right]}{\sqrt{ }\left(\phi_{s} \xi+q^{2}\right)}\right\} \\
\sim \int_{0}^{\xi} \frac{d \phi_{s}^{\prime}}{d \xi^{\prime}} \frac{d \xi^{\prime}}{\left\{1-\left(\xi^{\prime} \mid \xi\right)^{4 / 3}\right\}^{1 / 3}}
\end{gathered}
$$

where $\theta_{e}(\xi, 0)$ is given by

$$
\theta_{e}(\xi, 0)=\operatorname{Ln}\left\{\frac{4}{\phi_{s} \xi}\left(\phi_{s} \xi+q^{2}\right) \frac{\sqrt{ }\left(\phi_{s} \xi+q^{2}\right)+q}{\sqrt{ }\left(\phi_{s} \xi+q^{2}\right)-q}\right\}
$$

and $a$ is the reactant consumption parameter given by

$$
a=L_{e}^{2 / 3} \beta .
$$

This parameter $a$ is one of the most important parameters arising in this kind of problem. For small values of $a$, the reactant consumption does not play any role in the ignition process. When $a$ reaches unity it means that the temperature associated with the ignition is very close to the adiabatic fiame temperature. There is a critical value of $\alpha$, close to unity, for which the solution to the governing equations ceases to be multivalued. Thus, ignition cannot be characterized by a singular phenomenon like thermal runaway. For values of a larger than this critical value, we obtain a smooth transition from frozen to equilibrium flow.

\section{ZELDOVICH APPROXIMATION}

In a first approximation, neglecting terms of order $\varepsilon$, Eq. (38) has the solution

$$
q=-\sqrt{ }\left(1-\phi_{s} \xi\right)
$$




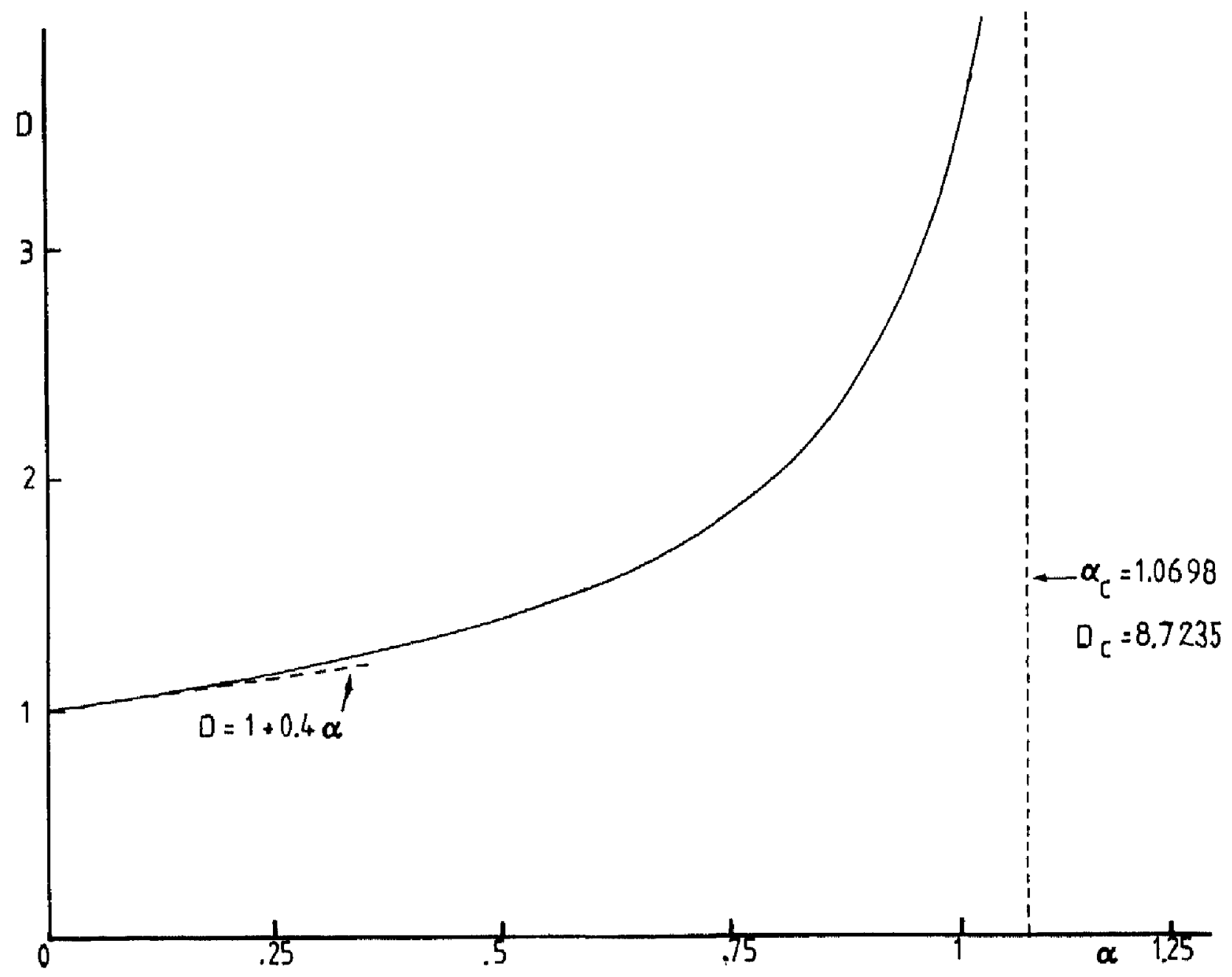

FIGURE 1 Critical Damköhler number for ignition $D_{0}$ as a function of the reactant consunption parameter $\alpha$, obtained with the Zeldovich approximation (zeroth-order solution).

while Eq. (39) takes the form

$$
-a D\left\{1-\sqrt{ }\left(1-\phi_{s} \xi\right)\right\}=\int_{0}^{\xi} \frac{d \phi_{s}^{\prime}}{d \xi^{\prime}} \frac{d \xi^{\prime}}{\left\{1-\left(\xi^{\prime} / \xi\right)^{3 / 4}\right\}^{1 / 3}} .
$$

Equation (43) gives the evolution of the fuel concentration as a function of the reactant consumption parameter $\alpha$.

Due to the fact that ignition (thermal runaway) can be assumed to occur at $\xi=1$, from Eq. (42) we have $\phi_{s}=1$ at ignition. Then $D$ is to be selected in such a way that $\phi_{s}=1$ coincides with $\xi=1$ in the solution to Eq. (43). Figure 1 shows the Damköhler number for ignition as a function of $a$. In the limit $a=0$, that is for very low plate temperatures, solution of Eq. (43) is given by $\phi_{s}=$ constant $=1$. Therefore $D(0)=1$ for this case. This result has been obtained by Berman and Ryazantsev (1978) and by Law and Law $(1979,1981)$. For small values of $a$, we can write

$$
Y=1-a \varphi_{s}(\xi)+O\left(a^{2}\right) \quad \text { as } \quad a \rightarrow 0,
$$


with the initial condition $\varphi_{s}(0)=0$. Therefore, we can obtain the following expan-
sions for $\phi_{s}$ and $D$ :

and

$$
\phi_{s}+1+a\left[\varphi_{s}(1)-\phi_{s}\right]+O\left(\alpha^{2}\right) \text { as } a \rightarrow 0
$$

$$
D=1+a \varphi_{s}(1)+O\left(\alpha^{2}\right) \text { as } a \rightarrow 0 .
$$

Introducing the relationships (45), (46) into the integral Eq. (43) we obtain to the lowest order in a:

$$
1-\sqrt{ }(1-\xi)=\int_{0}^{\xi} \frac{d \varphi_{s}^{\prime}}{d \xi^{\prime}} \frac{d \xi^{\prime}}{\left\{1-\left(\xi^{\prime} / \xi\right)^{3 / 4}\right\}^{1 / 3}},
$$

with the initial condition $\varphi_{s}(0)=0$. Equation (47) can be inverted (via Abel inversion) and the integral can be evaluated numerically giving $\varphi(1)=2 / 5$.

This solution (straight line) is plotted also in Figure 1. This gives very good results until values of $\alpha<0.3$. Increasing the value of $\alpha$ increases also drastically the Damköhler number for ignition. This does not mean that ignition is more difficult to achieve as $a$ increases as some authors believe. If $\alpha$ increases due to the increase of the temperature of the plate, the exponential term in the definition of the Damköhler number overcompensates the effect of the increase in $D$ with $\alpha$. There is a critical value of $a\left(a_{c}=1.0698\right)$ above which the singular behavior of the ignition phenomena disappears and a smooth transition from frozen to equilibrium flow is obtained. Therefore, ignition is not characterized by the thermal runaway. For this critical value of $a$, we obtain a finite Damköhler number for ignition, $D\left(a_{c}\right)=8.7235$. This is due to the convective effects (non-similar effects), that cause an increase in the fuel concentration from that given by the self-similar solution.

\section{IGNITION PROCESS FOR $\alpha<a_{0}$}

For values of $a<a_{0}$, the convective effects in the boundary layer have to be taken into account in order to obtain a better approximation for the ignition process. Close to the ignition point, $\xi \rightarrow 1$, the gradient of the apparent temperature is very important and has to be taken into account in order to determine the ignition distance. This means that the heat loss towards the boundary layer would increase drastically, thus causing a slower ignition process. Therefore, the correction to the values obtained by the Zeldovich approximation can be very important for finite values of the Zeldovich number. The derivative of the apparent temperature is given by

$$
\begin{aligned}
\frac{d \theta_{e}}{d \xi}= & \frac{2 \frac{d q}{d \xi}\left[q+\sqrt{ }\left(\phi_{s} \xi+q^{2}\right)\right]}{\phi_{s} \xi+q^{2}}-\frac{2}{\xi}+\frac{\phi_{s}}{\phi_{s} \xi+q^{2}}+\frac{\phi_{s}}{\sqrt{ }\left(\phi_{s} \xi+q^{2}\right)\left[\sqrt{ }\left(\phi_{s} \xi+q^{2}\right)+q\right]} \\
& +\left\{-\frac{2}{\phi_{s}}+\frac{\xi}{\phi_{s} \xi+q^{2}}+\frac{\xi\left(\phi_{s} \xi+q^{2}\right)^{-1 / 2}}{\left[\sqrt{ }\left(\phi_{s} \xi+q^{2}\right)+q\right]}\right\} \frac{d \phi_{s}}{d \xi} .
\end{aligned}
$$

The first te:m on the right-hand side of Eq. (48) shows a divergence close to the ignition. If we denote this function $F_{1}(\alpha, \xi)$ and the rest of the right-hand side as 
$F_{2}(\alpha, \xi)$ Eq. (38) can be rewritten as

$$
\begin{aligned}
& 1-\sqrt{ }\left(\phi_{s} \xi+q^{2}\right)+\varepsilon \xi \frac{\left[\phi_{s}-\frac{1}{2}\left(\phi_{1 p}+\frac{\gamma}{\varepsilon}+\frac{\gamma}{\varepsilon} q \int_{0}^{\infty} e^{2 /} d \chi\right)\right]}{\sqrt{ }\left(\phi_{s} \xi+q^{2}\right)} \\
& \sim-\frac{\varepsilon}{\lambda} \int_{0}^{\xi} \frac{F_{1}\left(\alpha, \xi^{\prime}\right) d \xi^{\prime}}{\left\{1-\left(\xi^{\prime} / \xi^{3 / 4}\right\}^{1 / 3}\right.}-\frac{\varepsilon}{\lambda} \int_{0}^{1} \frac{F_{2}\left(\alpha, \xi^{\prime}\right) d \xi^{\prime}}{\left\{1-\left(\xi^{\prime} / \xi\right)^{3 / 4}\right\}^{1 / 3}} \\
&+\frac{\varepsilon}{\lambda} \int_{\xi}^{1} \frac{F_{2}\left(\alpha, \xi^{\prime}\right) d \xi^{\prime}}{\left\{1-\left(\xi^{\prime} \mid \xi^{3 / 4}\right\}^{1 / 3}\right.} .
\end{aligned}
$$

The right-hand term is important only in regions close to the ignition $(\xi \rightarrow 1)$. How* ever, $F_{2}(a, \xi) \rightarrow 0$ as $\xi \rightarrow 1$. Then $F_{2}(a, \xi)$ can be obtained from the Zeldovich approximation:

$$
F_{2}(\xi)=\left[\sqrt{ }\left(1-\phi_{s} \xi\right)-\left(1-\phi_{s} \xi\right)\right]\left[\frac{1}{\xi}+\frac{1}{\phi_{s}} \frac{d \phi_{s}}{d \xi}\right] .
$$

The second integral can be evaluated numerically as

$$
I_{0}(\alpha)=\int_{0}^{1} \frac{F_{2}\left(\alpha, \xi^{\prime}\right) d \xi^{\prime}}{\left\{1-\left(\xi^{\prime} \mid \xi^{9 / 4}\right\}^{1 / 3}\right.} .
$$

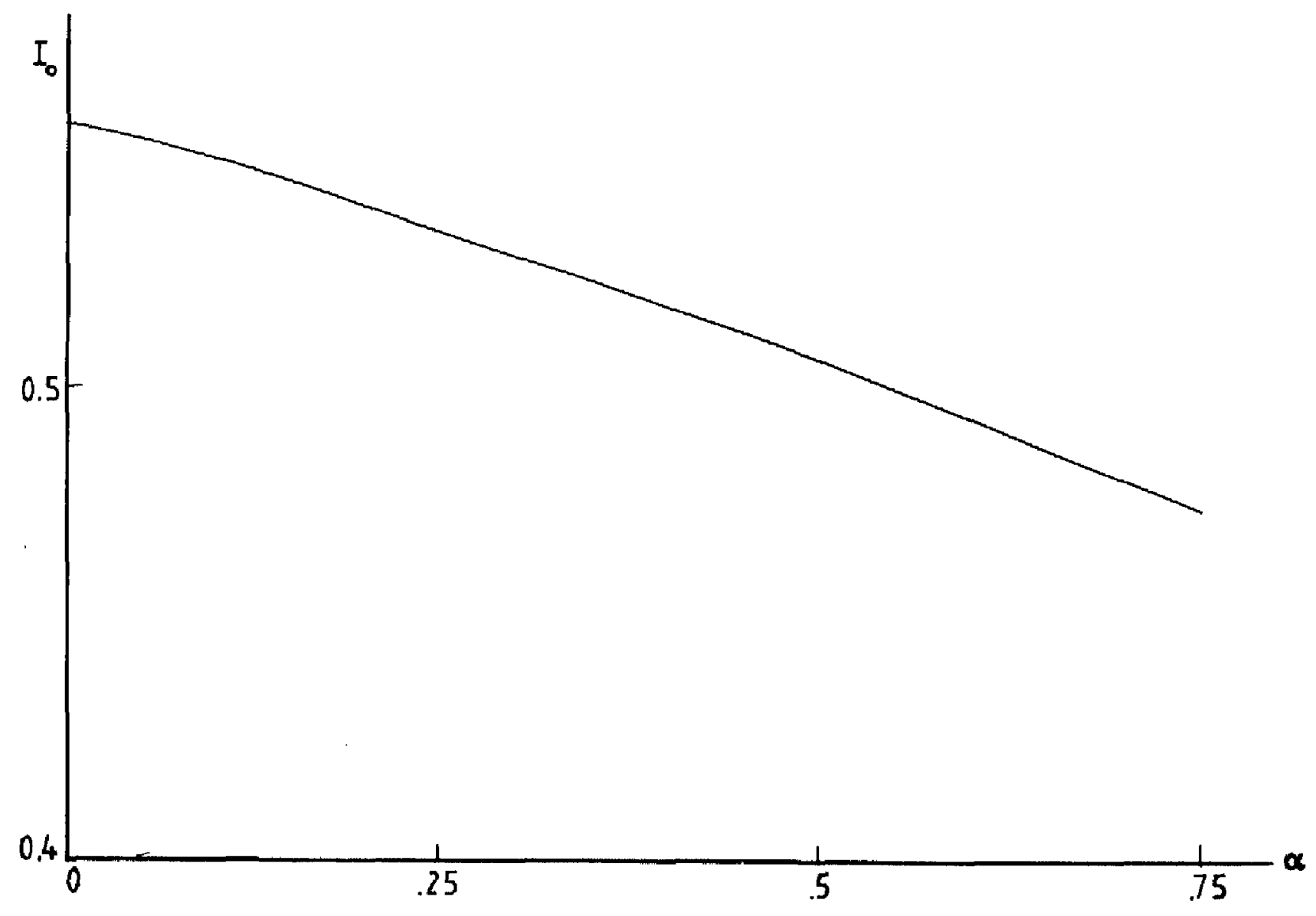

FIGURE 2 Definite integral $I_{0}$ as a function of the reactant consumption parameter $\alpha$. 


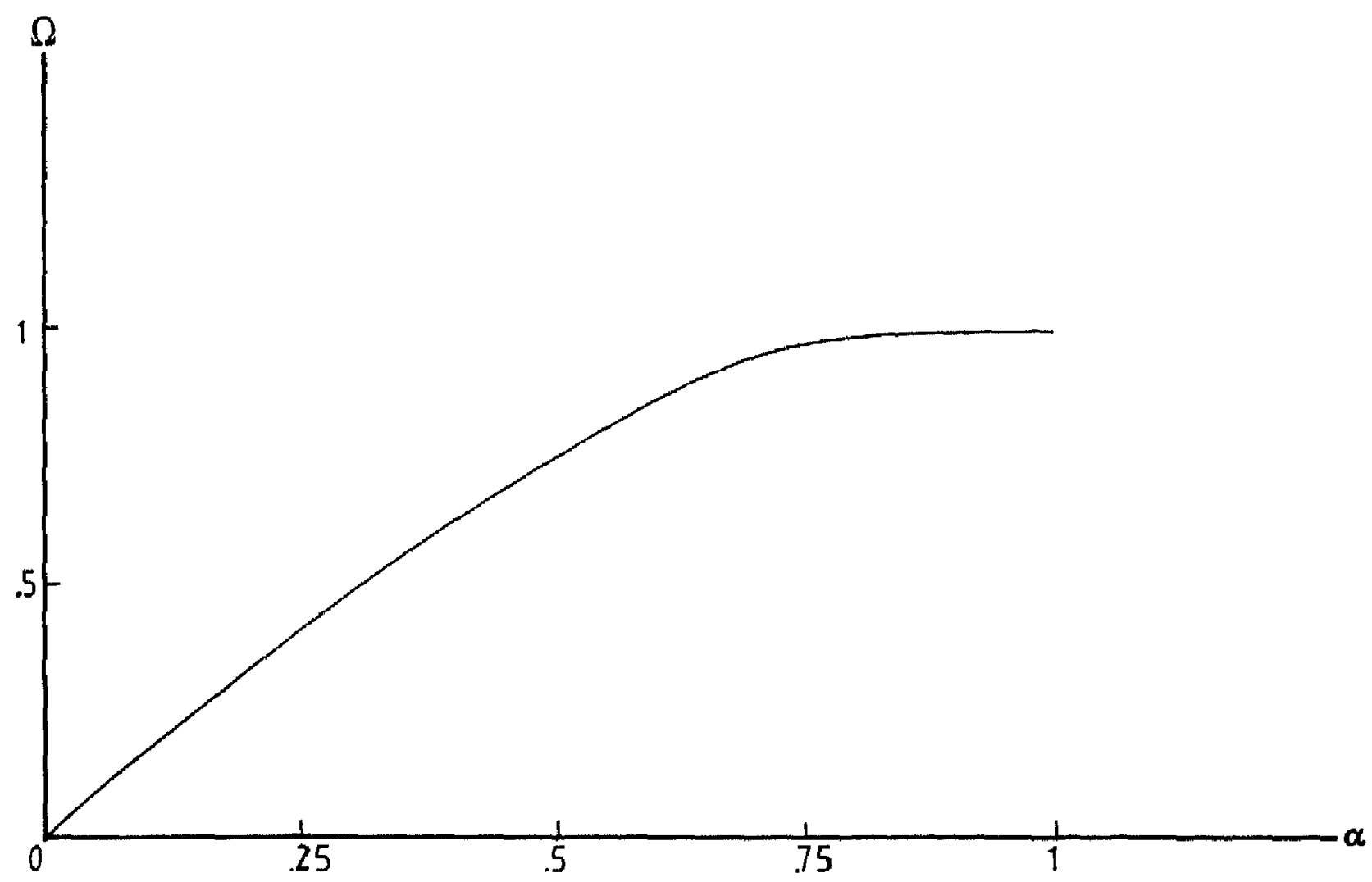

FIGURE 3 Parameter $\Omega$ as a function of the reactant consumption parameter $\alpha$.

This integral as a function of $\alpha$ is plotted in Figure 2. The numerical integration of Eq. (43) gives that for $\xi \rightarrow 1$

$$
\phi_{s} \sim \Omega(a)(1-\xi)+1 \text { as } \xi \rightarrow 1,
$$

where $\Omega(a)$ is plotted against $a$ in Figure 3. In order to obtain the correction to the Zeldovich approximation, we assume that

$$
D(a)=D_{0}(\alpha)[1+\delta(a)], \quad \delta(\alpha) \rightarrow 0 \text { for } \varepsilon \rightarrow 0,
$$

where $D_{0}(\alpha)$ is the Damköhler number obtained by the Zeldovich approximation. Then $\phi_{s}$, for $\xi \rightarrow 1$, is given by

$$
\phi_{s} \sim 1+\Omega(1-\xi)+\delta+O[\delta(1-\xi)] \quad \text { as } \quad \xi \rightarrow 1 .
$$

To study the behavior of the solution close to the ignition, we define the following stretching variables of order unity

$$
x=\frac{(1-\Omega)(1-\xi)}{M^{2} \varepsilon^{6 / 5}} .
$$


and

$$
y=\frac{q}{M \varepsilon^{3 / 5}},
$$

where

$$
M=\left[\left(\frac{4}{\lambda}\right)^{3}\left(\frac{4}{3}\right)(1-\Omega)\right]^{1 / 5} .
$$

Introducing the relations (55) and (56) into $\mathrm{Eq}$. (49) we obtain

$$
x-y^{2}-\Delta \sim \int_{x}^{1-\Omega / \varepsilon^{5 / s} M^{2}} \frac{d y^{\prime}}{d x^{\prime}} d x^{\prime} \frac{\left(1+\varepsilon^{3 / 5} M y^{\prime}\right)}{\left(x^{\prime}-x\right)^{1 / 3}}
$$

where $\Delta$ is given by

$$
\Delta=\frac{\delta-2 \varepsilon+\gamma(1+\ln 4)-\frac{\varepsilon}{\lambda} I_{0}(\alpha)}{\varepsilon^{6 / 5} M^{2}}
$$

For large values of $x$, the asymptotic solution to the integral equation (57) is that $y=-\sqrt{ } x$. Introduction of this relation in the integral term produces a divergence that we can correct to the lowest order by adding to both sides of the equation the following integral

$$
\frac{1}{2} \int_{x}^{1-\Omega / \varepsilon^{6 / 5} M^{2}} \frac{1}{\sqrt{ } x^{\prime}} \frac{d x^{\prime}}{\left(x^{\prime}-x\right)^{1 / 3}} \simeq \frac{3(1-\Omega)^{1 / 6}}{M^{1 / 3} \varepsilon^{1 / 5}}-2.5875 x^{1 / 6}+O(\varepsilon x) .
$$

Therefore, the integral equation (53) can be rewritten as

$$
x-y^{2}-\Delta+\frac{3(1-\Omega)^{1 / 6}}{M^{1 / 3} \varepsilon^{1 / 5}}-2.5875 x^{1 / 6} \sim \int_{x}^{1-\Omega / \varepsilon^{6 / 5} M^{2}}\left[\frac{d y^{\prime}}{d x^{\prime}}+\frac{1}{2 \sqrt{x^{\prime}}}\right] \frac{d x^{\prime}}{\left(x^{\prime}-x\right)^{1 / 3}} .
$$

The upper limit in the integral term of Eq. (60) can be moved to infinity with the inclusion on both sides of the following integral

$$
\frac{\varepsilon^{3 / 5} M}{2} \int_{x}^{1-\Omega / \varepsilon^{6 / 5} M^{2}} \frac{d x^{\prime}}{\left(x^{\prime}-x\right)^{1 / 3}}
$$

This is done in order to make it divergence-free at the upper limit. Therefore, Eq. (60) transforms to

$$
\begin{aligned}
x-y^{2}-\Delta+ & \frac{3(1-\Omega)^{1 / 6}}{M^{1 / 3} \varepsilon^{1 / 5}}\left[1-\frac{(1-\Omega)^{1 / 2}}{4}\right]-2.5875 x^{1 / 6} \\
& \sim \int_{x}^{\infty}\left[\frac{d y^{\prime}}{d x^{\prime}}+\frac{1}{2 \sqrt{ } x^{\prime}}\right] \frac{d x^{\prime}}{\left(x^{\prime}-x\right)^{1 / 3}} .
\end{aligned}
$$




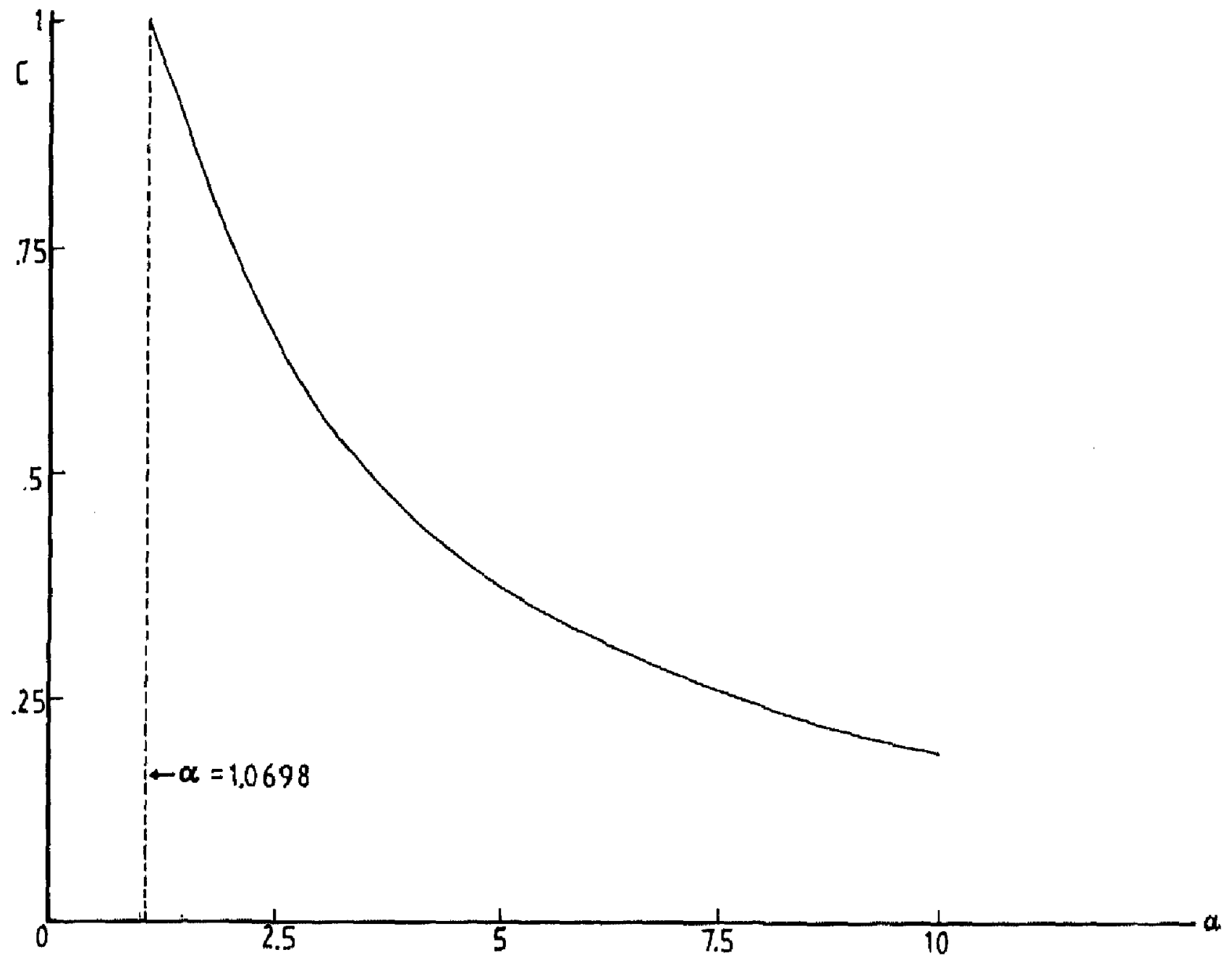

FIGURE 4 Parameter $C$ as a function of the reactant consumption parameter $\alpha_{\text {. }}$

Then $\Delta$ can be assumed to be given by

$$
\Delta=\frac{3(1-\Omega)^{1 / 6}}{M^{1 / 3} \varepsilon^{1 / 5}}\left[1-\frac{(1-\Omega)^{1 / 2}}{4}\right]+b+O\left(\varepsilon^{3 / 5}\right),
$$

where $b$ is a constant to be determined later. Then from Eqs. (58) and (63) we obtain the following expansion for $\delta$ :

$\delta=\left\{2+3(1-\Omega)^{1 / 6}\left[1-\frac{(1-\Omega)^{1 / 2}}{4}\right] M^{5 / 3}-\frac{\gamma}{\varepsilon}(1+\ln 4)+\frac{I_{0}}{\lambda}\right\} \varepsilon+b \varepsilon^{6 / 5} M^{2}+O\left(\varepsilon^{9 / 5}\right)$.

Therefore, Eq. (62) can be written as

$$
x-y^{2}-b-2.5875 x^{1 / 6} \sim \int_{x}^{\infty}\left[\frac{d y^{\prime}}{d x^{\prime}}+\frac{1}{2 \sqrt{ } x^{\prime}}\right] \frac{d x^{\prime}}{\left(x^{\prime}-x\right)^{2^{\prime \prime}}} .
$$

The solution to Eq. (65) gives the evolution of $y$ with $x$. This is a non-linear equation which admits a unique value of $b$ in order to have the divergence at $x=0$. The 
numerical calculation gives the value of $b=-1.125$. As $x$ approaches zero, the asymptotic behavior of the solution of Eq. (65) is given by

$$
y \sim \frac{k}{x^{1 / 3}} \text { as } x \rightarrow 0 .
$$

However, as $x$ becomes of order $\varepsilon^{9 / 5}$, the solution breaks down on one side because the non-dimensional heat flux, $q$, is no longer small compared with unity and on the other side the convective terms cannot be ignored.

Equation (66) dictates the matching to a transition stage which ends in the phenomenon called the explosion stage by Kapila $(1980,1981)$, which is convective limited rapid process. The analysis of this relatively complex phenomenon is left for future work.

\section{FLAME GENERATION WITH $a>a_{c}$}

For values of a larger than the critical one, there is a smooth transition from the nearly frozen flow to the deflagration wave. Therefore, in this case, the ignition is

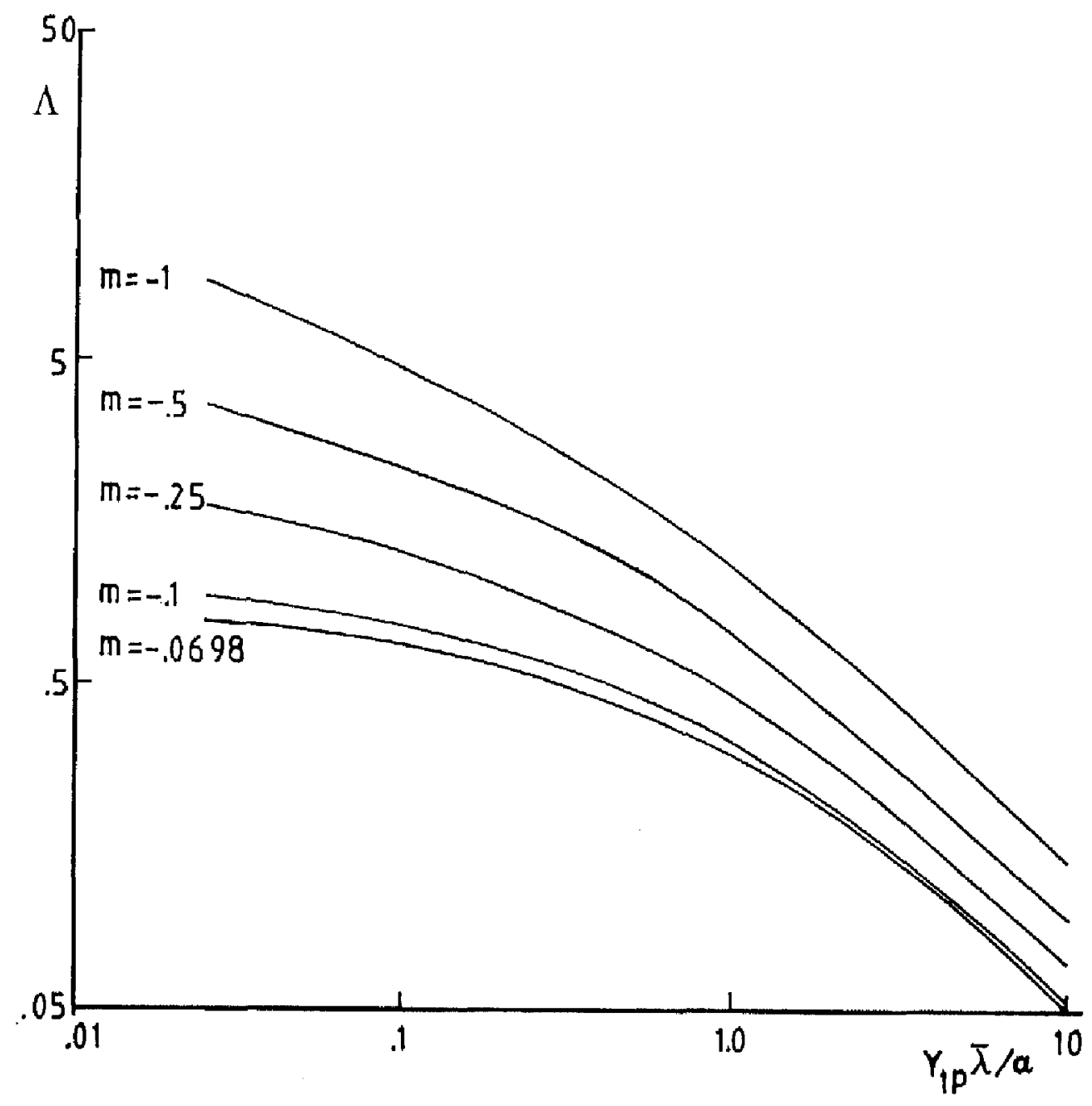

FIGURE 5 Non-dimensional length $\Lambda$ as a function of $Y_{1 F} \lambda / \alpha$, the reduced reactant concentration at the wall, for different values of the parameter $m(m=1-\alpha)$. 
not characterized by a thermal runaway. To the lowest order, the solution of the reactive zone equations can be obtained from Eqs. (42) and (43). The asymptotic behavior for large values of $\zeta$, where $\zeta=D \xi$, is given by

and

$$
q \sim-\sqrt{ }[1-C(a)]
$$

$$
Y \sim \frac{C(a)}{\zeta} \text { as } \zeta \rightarrow \infty .
$$

The function $C(a)$ is shown in Figure 4 and is obtained from the numerical solution of Eq. (43). However, this solution breaks down for values of $\zeta \sim \varepsilon^{-1}$. This is because the assumption of a uniform fuel concentration through the reactive zone is no longer valid. To study the further evolution of the premixed flame we define the following variables

and

$$
Y_{1}=\frac{Y}{\varepsilon}
$$

$$
\zeta=\varepsilon \zeta
$$

To the lowest order, the inner equations (21) and (22) then take the form

$$
\frac{\gamma^{2} \psi}{\partial X^{2}}=-\frac{\lambda}{a} \frac{\partial^{2} Y_{1}}{\partial X^{2}}=-\frac{\zeta Y_{1}}{2} \exp (\psi)
$$

where $\lambda=\lambda / L_{e}^{1 / 3}$. The boundary conditions at the plate are given by

$$
\psi=\frac{\partial Y_{1}}{\partial X}=0 \text { at } \chi=0 .
$$

Matching with the outer zone gives the boundary conditions for $\chi \rightarrow \infty$ as

$$
\frac{\partial \psi}{\partial X} \sim-1 \text { and } \frac{\partial Y_{1}}{\partial X} \sim \frac{1}{\lambda} .
$$

From Eq. (69), after integration once and applying the boundary conditions at the plate, we obtain

$$
\frac{\partial \psi}{\partial X}+\frac{\lambda}{a} \frac{\partial Y_{1}}{\partial X}=q
$$

and with the aid of the relations given in (71) we have it that

$$
q=\frac{1-\alpha}{\alpha}
$$

thus, the heat flux from the plate can be assumed to be constant and negative. That is, the temperature of the plate is higher than the adiabatic flame temperature.

Integration of Eq. (72) gives

where

$$
\begin{aligned}
\psi & =q X-r, \\
r & =\frac{\lambda}{a}\left(Y_{1}-Y_{1 p}\right) .
\end{aligned}
$$




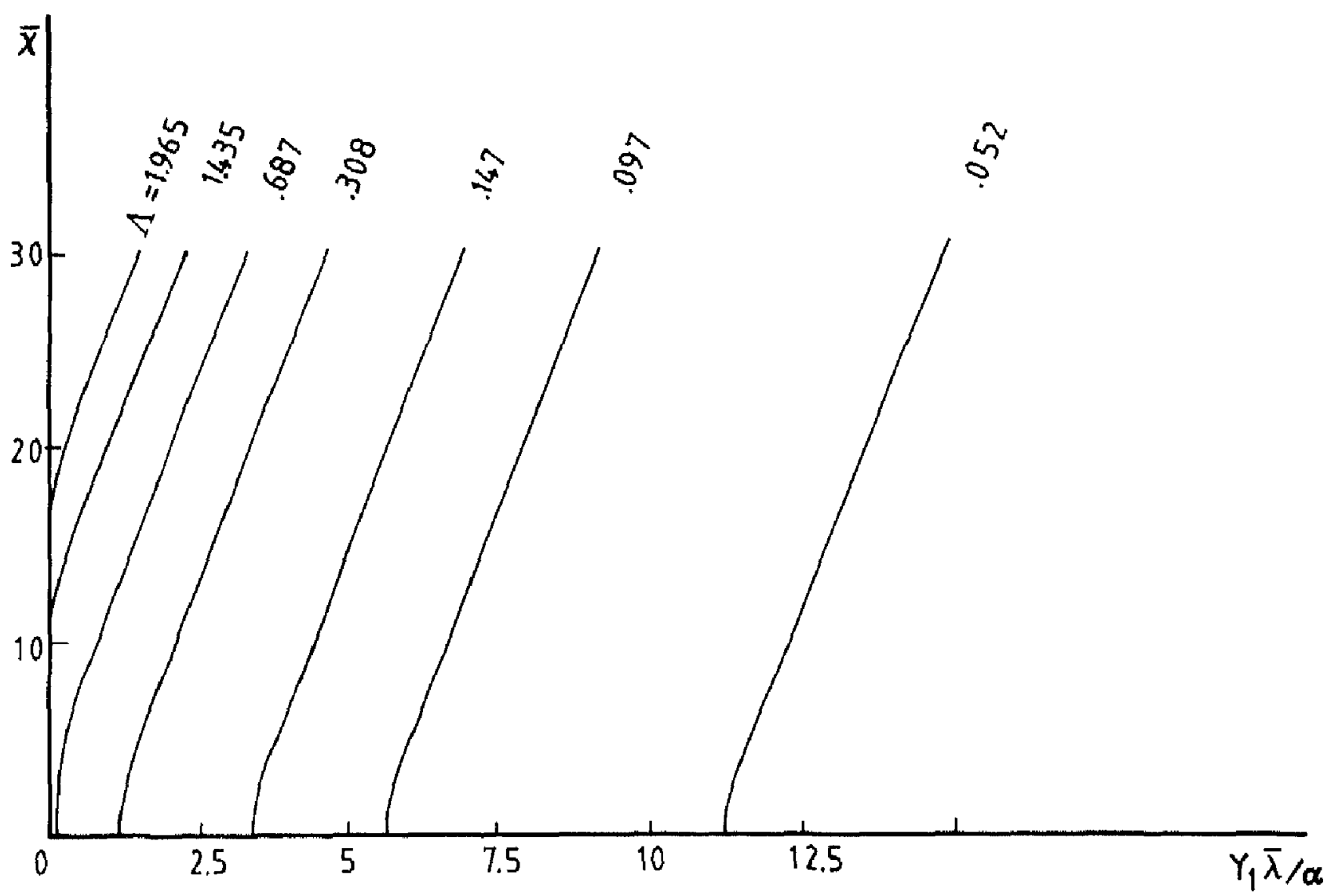

FIGURE 6 Reduced reactant concentration $Y_{1} \delta / \alpha$ profiles as a function of the non-dimensional transversal distance $\bar{\chi}$, for different values of the non-dimensional length $\Lambda$ and $m=-0.0698$.

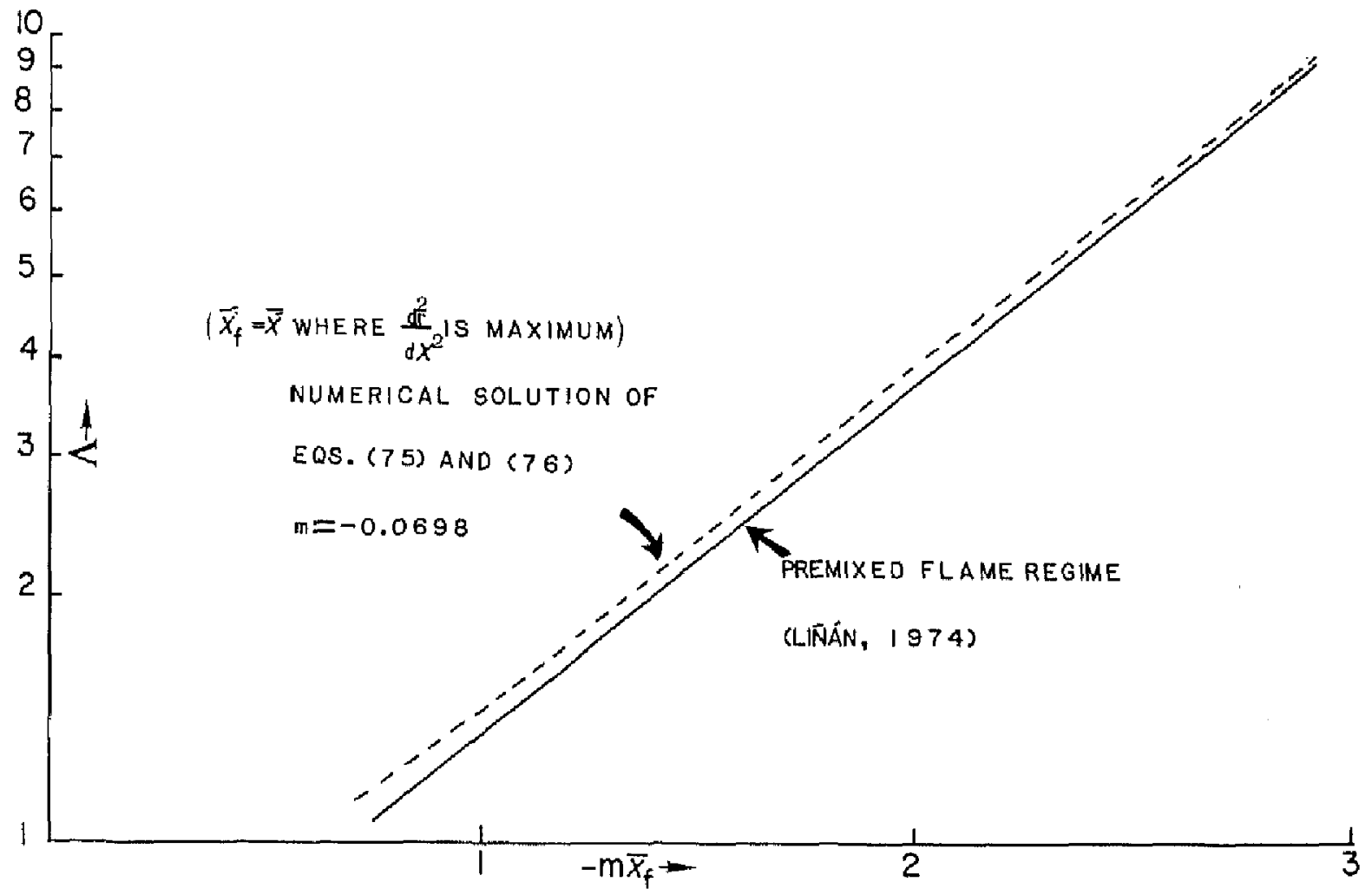

FIGURE 7 Premixed flame position $\bar{x} s$ as a function of the non-dimensional length $A$, for different values of the parameter $m$. As a comparison, the results of Liñan are shown. 
$Y_{1 p}$ being the value of $Y_{1}$ at the surface. Equation (69) can be rewritten as

$$
\frac{\partial^{2} r}{\partial \bar{\chi}^{2}}=\Lambda\left(r+\frac{\lambda Y_{1 p}}{a}\right) \exp (-r+m \bar{\chi}),
$$

with

and

$$
\begin{aligned}
& \bar{\chi}=x / a \\
& m=1-a
\end{aligned}
$$

$$
\Lambda=\frac{\xi \alpha^{3}}{2 \bar{\lambda}} .
$$

The boundary conditions for Eq. (75) are the following:

and

$$
r=\frac{\partial r}{\partial \bar{X}}=0 \quad \text { at } \quad \bar{\chi}=0
$$

$$
\frac{\partial r}{\partial \bar{x}}=1 \quad \text { at } \quad \bar{x} \rightarrow \infty .
$$

This represents an eigenvalue problem having the solution for a given value of $\Lambda$,

$$
\Lambda=\Lambda\left(\alpha, \lambda, Y_{1 p}\right)
$$

This relation is plotted in Figure 5 for different values of $m$. The fuel concentration profiles are plotted in Figure 6 as a function of $\ddot{\chi}$ for different values of the reduced longitudinal coordinate $\Lambda$. It can be observed that for large values of $\Lambda$, a thin reactive zone is located at a well-defined $\bar{x}_{f}(\Lambda)$ which separates the frozen region from the equilibrium region.

The asymptotic solution obtained by Liñán (1974) for the premixed flame regime can be used in order to follow the further evolution of the premixed flame. We introduce

Thus, Eq. (75) reduces to

$$
\sigma=\bar{\chi}_{f}-\bar{\chi} .
$$

$$
\frac{\partial^{2} r}{\partial \sigma^{2}}=\left[\Lambda \exp \left(m \bar{\chi}_{f}\right)\right] r \exp (-r-m \sigma),
$$

with the boundary conditions

and

$$
\frac{\partial r}{\partial \sigma}=0 \quad \text { at } \quad \sigma=\bar{\chi}_{f} \rightarrow \infty
$$

$$
\frac{\partial r}{\partial \sigma}=1 \quad \text { at } \quad \sigma \rightarrow-\infty .
$$

Equations (78) and (79) have a solution only for

$$
\Lambda \exp \left(m \overline{\chi_{s}}\right)=\frac{1}{2},
$$


which gives the relationship between $\bar{\chi}_{f}$ and $\Lambda$. That is, it gives the position of the premixed flame in the $(\bar{x}, \Lambda)$ plane. This relation is plotted in Figure 7 as compared with the numerical solution to Eqs. (75) and (76). The flame non-dimensional temperature can be obtained through the following relation obtained by Liñan (1974):

$$
\psi_{f}=\theta_{f}-\chi_{f}=-p_{0}+m \bar{\chi}_{f},
$$

where $p_{0}$ is a function of $a$ (or $m$ ) and can be obtained from the correlation given by Liñán (1974),

$$
p_{0}=-\frac{1}{m} \operatorname{Ln}\left[0.6307 m^{2}-1.344 m+1\right] .
$$

Therefore, Eq. (81) shows that the non-dimensional flame temperature decreases as the flame separates from the wall due to the fact that $m$ is negative in this regime.

\section{RESULTS AND CONCLUSIONS}

In this paper an analysis is made on the premixed combustion of combustible gases ignited by a hot flat plate in a boundary layer flow. One of the most important parameters in this type of process is the reactant consumption parameter $a$. This parameter measures the effect of the fuel consumption in the ignition process. For small values of this parameter (low temperatures of the plate), the rapid increases in the gas temperature take place without fuel consumption. For values of a less than a critical value $\left(a_{c}=1,0698\right)$ ignition is characterized by a thermal runaway. In this case, a three-term asymptotic series is obtained for the Damköhler number for ignition that gives very good results for moderate values of the Zeldovich number. In Table I a comparison is made between results obtained through numerical integration of the governing equations by Law and Law (1979) and that obtained analytically in this work.

TABLE I

Comparison between analytical solution $D$ and the numerical results $D_{n}$ for the critical Damköhler number for ignition

\begin{tabular}{lllcc}
\hline \multicolumn{1}{c}{$\varepsilon$} & \multicolumn{1}{c}{$\alpha$} & $\lambda$ & $D_{n}$ (numerical) & $D$ (analytical) \\
\hline 0.1 & 0.0875 & 0.7 & 2.0861 & 2.0366 \\
0.102 & 0.09 & 0.706 & 2.1095 & 2.0467 \\
0.1 & 0.075 & 0.6 & 2.1794 & 2.1366 \\
0.1 & 0.175 & 0.7 & 2.2861 & 2.1775 \\
0.10204 & 0.0875 & 0.7 & 2.0973 & 2.0536 \\
\hline
\end{tabular}

This table shows a very good agreement within a few percent. However, the effect of the Zeldovich number is important as shown in comparison with the several terms in the asymptotic series. As mentioned before, this is due to the higher order effects in the convective heat transfer rates towards the outer non-reactive zone. The critical dimensional distance from the leading edge in order to achieve the ignition condition 
for values of $a<a_{c}$ is then given by

$$
L_{I}=\frac{u_{\infty}\left[\beta f^{\prime \prime}(0)\right]^{2}}{2 \bar{B} P r^{1 / 3} \varepsilon} \exp \left(\frac{\bar{T}_{a}}{\bar{T}_{p}}\right) D_{0}(a)\left[1+J_{1} \varepsilon+J_{2} \varepsilon^{6 / 5}+O\left(\varepsilon^{9 / 5}\right)\right]
$$

with

$$
\begin{aligned}
& J_{1}=2+3(1-\Omega)^{1 / 6}\left[1-\frac{(1-\Omega)^{1 / 2}}{4}\right] M^{5 / 3}-\frac{\gamma}{\varepsilon}(1+\ln 4)+\frac{I_{0}(\alpha)}{\lambda}, \\
& J_{2}=-1.125 M^{2},
\end{aligned}
$$

and

$$
M=\left[\left(\frac{4}{\lambda}\right)^{3}\left(\begin{array}{l}
4 \\
3
\end{array}\right)(1-\Omega)\right]^{1 / 5}
$$

For values of a larger than $\alpha_{c}$, the further evolution of the process leading to a premixed fiame is studied. The location of this flame was obtained and the flame temperature was deduced, showing a decrease as the flame separates from the wall. This is because in this regime, the plate temperature is higher than the adiabatic flame temperature of the combustible mixture. The results obtained by Liñan (1974) for his premixed fiame regime, are used in order to study the evolution of the premixed flame as it is located far away from the wall.

\section{Appendix}

\section{IGNITION ENERGY}

In this paper we have assumed a uniform temperature of the plate. However, in order to achieve a certain temperature we have to add energy externally and this energy is called the ignition energy.

The results obtained in this work can be easily extended to the case where the thermal conductivity of the plate is high enough. We consider here a flat plate heated from below with the average heat flux per unit length and unit time denoted as $\bar{q}_{c}$. The local heat flux in the upper surface of the plate, $\bar{q}_{u}$, is related to the nondimensional heat flux $q$, defined in Eq. (30) as,

$$
\bar{q}_{u}=\frac{Q \bar{Y}_{F \infty}}{P r^{2 / 3}} \sqrt{\left(\frac{u_{\infty} p_{\infty} \mu_{\infty}}{2 L}\right) \beta f^{\prime \prime}(0) \frac{q}{\sqrt{\xi}} .}
$$

The steady-state condition can be achieved if the net heat flux at the surface vanishes. That is, the total heat flux in the upper surface equals the flux in the lower surface. This is expressed as

$$
\int_{0}^{L} \bar{q}_{u} d x=\bar{q}_{e} L
$$




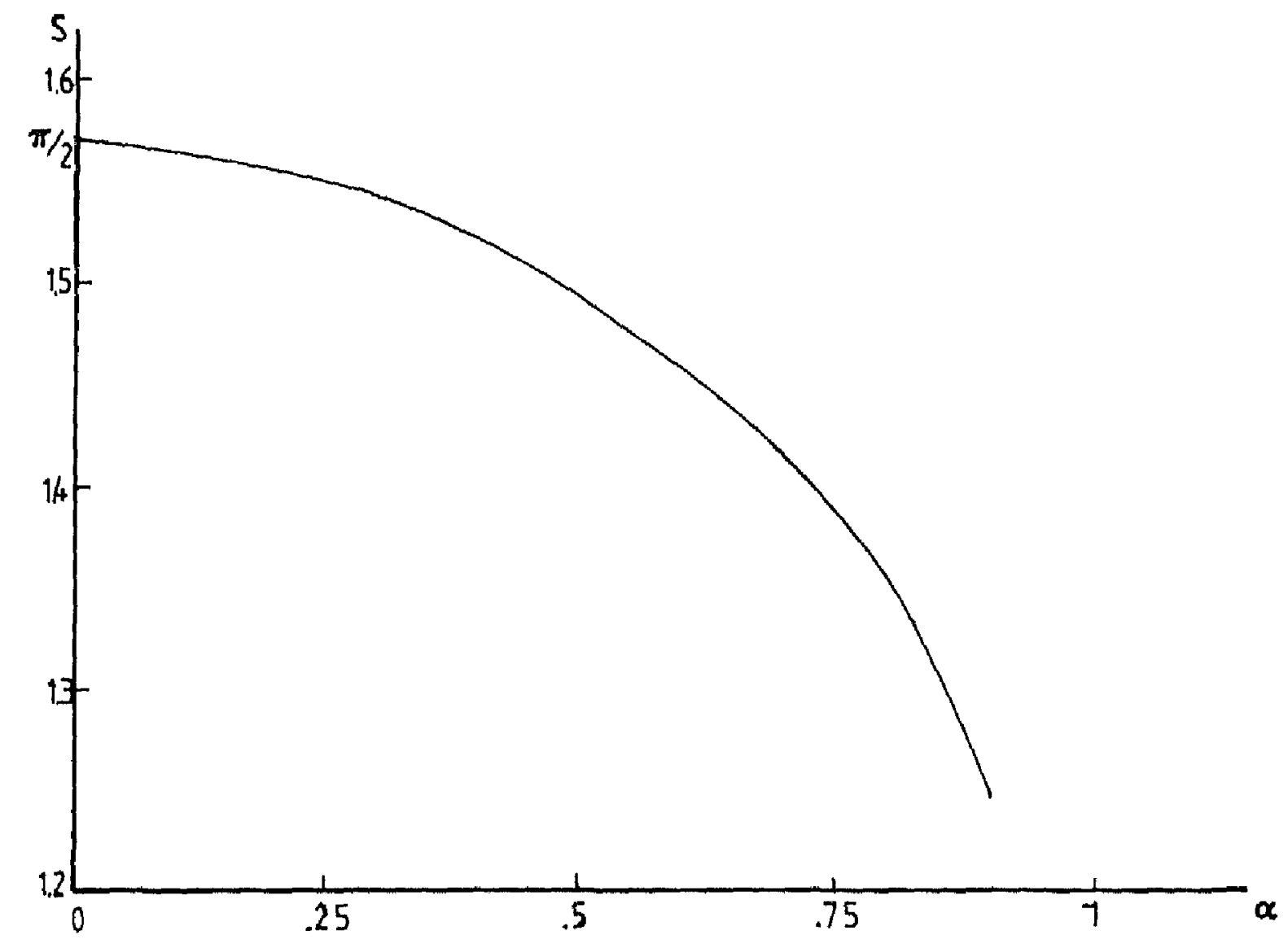

FIGURE A.1 Non-dimensional total heat-flux parameter $S$ as a function of the reactant consumption parameter $\alpha$.

Introducing Eq. (A.1) into Eq. (A.2) we obtain

$$
\int_{0}^{1} \frac{q}{\sqrt{ } \xi} d \xi=-\frac{\bar{q}_{e} P r^{2 / 3} \sqrt{ }\left(2 L^{3}\right)}{Q \bar{Y}_{F \infty} \sqrt{ }\left(u_{\infty} \rho_{\infty} \mu_{\infty}\right) \beta f^{\prime \prime}(0)} .
$$

Equation (A.3) gives the relationship of the temperature of the plate, included in $\beta$ and the external energy flux, $\bar{q}_{e}$. The left-hand side of Eq. (A.3) to the lowest order can be given by the Zeldovich approximation

$$
-\int_{0}^{1} \frac{\sqrt{ }(1-\phi \xi)}{\sqrt{ } \xi} d \xi=-S(\alpha)
$$

which is a function of the reactant consumption parameter $a$. This function is plotted in Figure (A.1). For $a=0$, from Eq. (A.4), it can be shown that $S(0)=\pi / 2$. From the definition of a given by Eq. (41), we obtain finally

$$
a S(a)=\frac{\bar{q}_{e} S c^{2 / 3} \sqrt{ }\left(2 L^{3}\right)}{Q \bar{Y}_{F_{\infty}} \sqrt{ }\left(u_{\infty} p_{\infty} \mu_{\infty}\right) f^{\prime \prime}(0)},
$$

which related the external energy flux with the reactant consumption parameter. The temperature of the plate can be obtained through Eqs. (13) and (41). 